\title{
PREDICTION OF THE SEASONAL CHANGES OF THE CHLORIDE CONCENTRATIONS IN URBAN WATER RESERVOIR
}

\author{
PROGNOZOWANIE SEZONOWYCH ZMIAN STĘ̇̇ENIA CHLORKÓW \\ W MIEJSKIM ZBIORNIKU WODNYM
}

\begin{abstract}
This study investigated the possibility of using artificial neural networks to predict changes in the concentration of chloride ions in the urban ponds on the example of the inflow and outflow zones of water to and from the ponds Syrenie Stawy in Szczecin (NW-Poland). The possibility of using selected water quality indices (selected based on correlation matrix of water quality indices with $\mathrm{Cl}^{-}$), in particular: COD-Cr, $\mathrm{BOD}_{5}$, DO, water saturation by $\mathrm{O}_{2}$ and $\mathrm{NO}_{2}{ }^{-}$and their influence on the chloride concentration forecast was tested.
\end{abstract}

Keywords: urban lake, water quality prediction, artificial neural network

\section{Introduction}

The protection of water quality in urban water bodies should be one of the most important tasks in environmental programs undertaken by municipalities in each city. Lakes, river ponds and streams, especially in larger cities, play an important role in recreation and tourism, and are often very often occupied by valuable biocoenoses [1-4].

Unfortunately, urban water bodies are collectors of pollution discharged into municipal and industrial sewage, leading to the degradation of these ecosystems. The changes in the water's biotope of such tanks are primarily the increase of dissolved concentrations and the involved organic matter and the change of mineralization, both qualitative and quantitative. One of the indicators investigated for the assessment of water quality, and especially for the study of changes in their mineralization, is the concentration of chloride ions [1-5].

The increase in salinity of water characterized by the increase in chloride ions concentrations signals changes in the quality of the biotope and the risks of biocenosis settling such reservoirs [5-15].

It is true that in cities located in the coastal zone, the increase in surface chlorine concentrations may be related to the deposition of sea water aerosols and to the washout of salt from the soil by rain. Due to the fact that urban water reservoirs are very often included

\footnotetext{
${ }^{1}$ Department of Chemistry and Natural Waters Management, Institute for Research on Biodiversity, Faculty of Biology, Szczecin University, ul. Felczaka 3C, 71-412 Szczecin, Poland, email: polesz@univ.szczecin.pl

* Corresponding author: tymoteusz.miller@usz.edu.pl
} 
in the rain drainage network in cities in temperate zone, where during the winter months $\mathrm{NaCl}$ and/or $\mathrm{CaCl}_{2}$ is used to remove glazed frost. In the periods of thaws, especially in early spring, when melting snow and ice cover of city streets flow into the tank substantial amounts of chloride ions. In this case the changes in chloride concentrations are seasonal [5-15]. Generally, in recent years we have seen an increase in the concentration of chloride ions in freshwater bodies of water around the world [5-15].

However, examination of the changes in concentrations of chloride ions in freshwater ecosystems in urban areas is a convenient way to check the nature of the changes of biotopes of examined water bodies [5-15].

Because urban water quality is often examined only occasional it seems to be justified using modern techniques which allows to discern and make predictions based on a limited number of measurements, which seems to be of particular importance for the study of changes in the quality of the water environment in cities.

One of the tools for predicting and modeling is artificial neural networks (ANN) a specialized mathematical "cause-effect" system, in its structure resembling a simple network of biological neurons. At first, artificial neural networks were used in economic sciences [16] and in meteorology [17]. Currently, more and more often used in prediction of changes in surface waters, demersal waters and hydrological changes [18-28].

There were studies using ANN to predict and determine the impact of various factors, such as catchment area, environmental or atmospheric factors - on the salinity level of marine, fresh or groundwater [29-32].

The aim of this work was to demonstrate the use of artificial neural networks for the forecasting of chloride ion concentrations in surface water in Ponds Syrenie Stawy in Szczecin using a relatively small number of measured data (20 measurements in subsequent months).

\section{Lake characteristic}

Ponds Syrenie Stawy in Szczecin are four artificial flow water reservoirs (Table 1, Fig. 1) formed in the second half of the 19th century after damming the waters of the Osowka stream. The Ponds are located in the Arkonski Forest, which is part of the protected nature and landscape complex "Kasprowicz-Arkonski Park Complex". The total area of Syrenie Stawy is about 1.99 ha, with an average depth of about $2 \mathrm{~m}$. Syrenie Stawy like others urban water bodies are included into municipal rain drainage system, where they play the role of reservoirs for precipitation waters as well as of settling ponds and biological treatment plants [33-36].

By the beginning of the 90s of the last century, significant amounts of various pollutants, including urban waste water and industrial waste water, were discharged into the Syrenie Stawy waters e.g. from POLMO Workshops and military unit workshops. This state of affairs was about to change after the sewage network was completed. After a gradual reduction of pollutant inflow with Osowka stream waters which flows through Ponds Syrenie Stawy the entire ecosystem of Ponds was gradually self purificating, which was interrupted in August - November 2003 by hydrotechnical works to regulate the runoff of the Osowka by constructing a new trough of Osowka, and then in period from December 2010 to December 2012, when a large-scale hydrotechnical works had place - changing the riverbed leading waters of Osowka and regulating the flow of water through the Ponds Syrenie Stawy which caused a negative change in the quality of ponds in subsequent years 
after these regulations. Since 2010, preparations are being made for the construction of the Botanical Garden - Arboretum "Syrenie Stawy" [33-35].

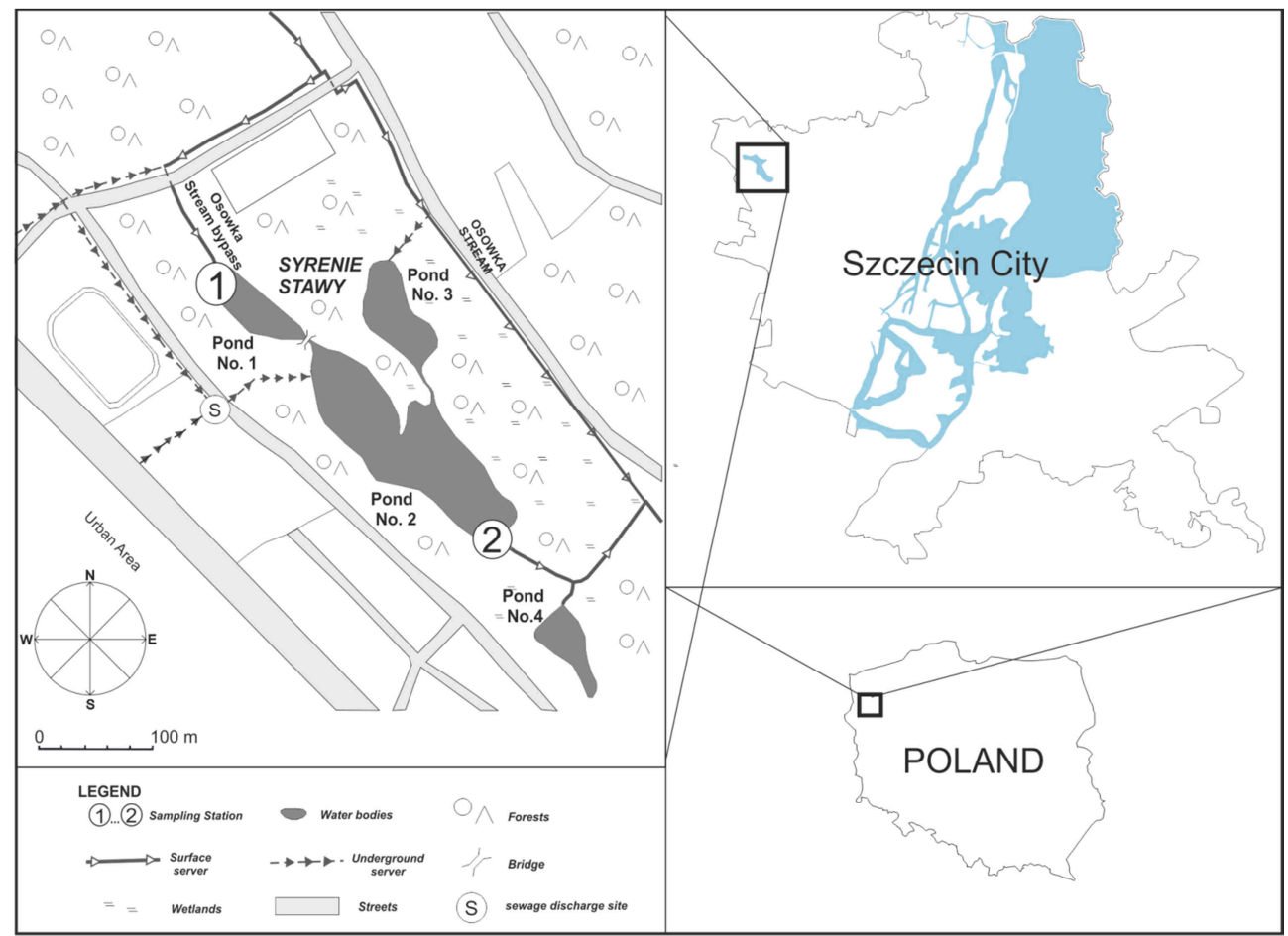

Fig. 1. Syrenie Stawy Ponds in Szczecin city (NW-Poland) (after: [33] with changes)

Morphometric characteristic of Syrenie Stawy Ponds

Table 1

\begin{tabular}{|c|c|c|c|c|c|}
\hline \multirow{2}{*}{ Geographical coordinates } & \multicolumn{2}{|c|}{ Latitude } & \multicolumn{3}{|c|}{$53^{\circ} 45^{\prime} 69.4^{\prime \prime} \mathrm{N}$} \\
\hline & \multicolumn{2}{|c|}{ Longitude } & \multicolumn{3}{|c|}{$14^{\circ} 51^{\prime} 05.1^{\prime \prime} \mathrm{E}$} \\
\hline \multicolumn{6}{|c|}{ Morphometric data } \\
\hline \multirow{2}{*}{ Morphometric indicator } & \multirow{2}{*}{ Units } & \multicolumn{4}{|c|}{ Pond No. } \\
\hline & & $\mathbf{1}$ & 2 & 3 & 4 \\
\hline Water level & {$\left[\mathrm{m} \mathrm{asl}^{*}\right]$} & 21.1 & 21.0 & 21.0 & 20.0 \\
\hline Area & {$\left[10^{4} \mathrm{~m}^{2}\right]$} & 0.27 & 1.15 & 0.34 & 0.13 \\
\hline Capacity & {$\left[10^{3} \mathrm{~m}^{3}\right]$} & 4.0 & 23 & 6.8 & 0.7 \\
\hline Depth - max & {$[\mathrm{m}]$} & 1.8 & 2.5 & 2.4 & 0.6 \\
\hline Depth - average & [m] & 1.5 & 2.0 & 2.0 & 0.5 \\
\hline Length max & [m] & 86 & 217 & 97 & 77 \\
\hline Width max & {$[\mathrm{m}]$} & 36 & 80 & 37 & 26 \\
\hline Length of coastline & [m] & 216 & 565 & 250 & 195 \\
\hline
\end{tabular}

${ }^{*}$ meters above sea level

After performing hydrotechnical works Syrenie Stawy act as retention tanks on the bypass passage of the Osowka watercourse. It had significantly contributed to the stabilization of the flow rate of this stream. As a result, the retention time of ponds is 
variable - during intense and prolonged precipitation it reaches up to 14 days, and in periods when precipitation does not occur - water of Ponds stagnate. So Ponds Syrenie Stawy from the hydrological point of view are overflowed Osowka [33, 34].

\section{Material and methods}

The water samples of Ponds Syrenie Stawy were collected for examination at the place of two sampling stations located respectively in the region of inflow of water to the Pond No. 1 and the water outflow area of the Pond No. 2 - which is the main flow path of water by Syrenie Stawy. Surface water samples were taken from a depth of c.a. $25 \mathrm{~cm}$ at 30-day intervals from January 2014 to August 2015. 19 selected water quality indices, in particular temperature, $\mathrm{pH}$, chemical oxygen demand as COD-Cr and COD-Mn, dissolved oxygen (DO), water saturation by $\mathrm{O}_{2}$ (WS), 5-day biochemical oxygen demand $\left(\mathrm{BOD}_{5}\right.$ ), concentration of $\mathrm{NO}_{3}^{-}, \mathrm{NO}_{2}^{-}, \mathrm{NH}_{4}{ }^{+}, \mathrm{PO}_{4}{ }^{3-}$, total concentrations of nitrogen (TN) and phosphorus (TP), total hardness $(\mathrm{TH})$, concentration of calcium $\left(\mathrm{Ca}^{2+}\right)$, sulfate $\left(\mathrm{SO}_{4}{ }^{2-}\right)$, chloride $\left(\mathrm{Cl}^{-}\right)$, alkalinity (ALK) and total concentrations of iron $\left(\mathrm{Fe}_{\mathrm{tot}}\right)$. Samples methods as well as all analyzes were performed according to recommendations [37].

\section{Artificial neural network (ANN)}

Artificial neural networks are considered nonlinear statistical tools for modeling and forecasting data variability. It is used when standard statistical and mathematical procedures do not provide a satisfactory answer when solving a problem [38-46].

ANNs are groups of interconnected artificial neurons using a mathematical model for processing information based on a "connection" computational approach. This is an adaptive system that changes its structure according to the data flowing to and through the system network [38-46].

The basic unit of artificial neural networks are nodes. They are connected by the so-called synapses which are related with appropriate weighting factors. The most commonly used network model is the three-layer ANN model, in which we distinguish the input layer, hidden layer and output layer [38-46].

In order to produce mathematical models with ANN, it is necessary to introduce so-called typical data pairs - to generate a training set. An appropriately trained network will test its knowledge of a given problem by creating a testing set and then validating the set [38-46].

In order to develop an automatic neural network that predicts chloride ion concentration in incoming and outgoing water to/from Syrenie Stawy, the data set was divided into three sets: $60 \%$ for the learning set and $20 \%$ for the test and validation set. To define the final model, time series regression analysis was performed using the multilayer perceptron (MLP) ANN model with the maximum number of hidden layers defined as 10, and linear, logistic, tanh, exponential, and sinusoidal functions were used as activation functions for hidden and output neurons [38-46].

\section{Model performance indicators}

In this study, the results obtained using ANN were compared and evaluated [44-51]. It is important to define average forecast error by $(M S E, R M S E)$, model fit $\left(R^{2}\right)$, and prognostic error distribution when creating forecast models. The robustness of the model 
was tested by determining the mean absolute error of the prediction (MAPE) - defining the percent precision of the model according to the formula [44-51]:

$$
M A P E=\frac{100}{n} \sum_{i=1}^{n}\left|\frac{x_{i}-p_{i}}{x_{i}}\right|
$$

where $x_{i}$ is the measured chloride ion concentrations in $i$-th month, $p_{i}$ - calculated with ANN values of chlorides in $i$-th month, $n$ - is the total number of observations.

As a criterion for determining the model performance results obtained by the ANN the coefficient of determination $\left(R^{2}\right)$ was calculated, defined as $[18,19,44-51]$ :

$$
R^{2}=\frac{\sum_{i=1}^{n}\left(p_{i}-\bar{x}\right)^{2}}{\sum_{i=1}^{n}\left(x_{i}-\bar{x}\right)^{2}}
$$

To determine the value of the prognostic error mean square error (MSE) and the root mean square error (RMSE) were calculated by the formulas:

$$
\begin{aligned}
M S E & =\frac{1}{n} \sum_{i=1}^{n}\left(x_{i}-p_{i}\right)^{2} \\
R M S E & =\sqrt{\frac{1}{n} \sum_{i=1}^{n}\left(x_{i}-p_{i}\right)^{2}}
\end{aligned}
$$

In addition, the discrepancy ratio for the obtained data (ANN output) was defined as [49-51]:

$$
D_{r}=\frac{p_{i}}{x_{i}}
$$

In order to avoid the multidimensional scattering of measured data, the data standardization was performed on the basis of equation [44, 45]:

$$
x_{s}=\frac{x_{i}-\bar{x}}{S D}
$$

where: $x_{s}$ - represents a standardized value, $x_{i}$ - value of the parameter being standardized, $\bar{x}$ - average value within the given parameter water quality, and $S D$ - standard deviation.

All calculations were performed using computer software STATISCITA 12.0PL.

\section{Results and discussion}

The results of the 19 indicators of water quality at the inflow and inlet from the Syrenie Stawy are presented in Table 2 and Figures 2 and 3. Variability of most of the indices was very similar in nature at both sampling stations. In the zone of water inflow to the Syrenie Stawy higher values - in relation to the outflow zone of the Ponds had COD-Cr, $\mathrm{NO}_{2}{ }^{-}, \mathrm{TN}$, SRP, $\mathrm{Cl}^{-}$, and $\mathrm{Fe}_{\text {tot }}$ at the same time with worse aerobic conditions (WS, DO) and lower $\mathrm{pH}$ values. This shows the influx of pollutants into the Ponds primarily with the waters of Osowka.

During the flow of water through the Syrenie Stawy in the study period, all changes in the values of the sedimentation indices are related to changes in the indices of organic matter sedimentation to bottom sediments and to the occurrence of relatively intensive 
oxidation processes of organic matter $(\mathrm{OM})$. This suggests that the Ponds Syrenie Stawy in the study period were relatively well-functioning reservoirs - biological treatment plants.

Table 2

Statistical characteristics of selected water indices in Ponds Syrenie Stawy

\begin{tabular}{|c|c|c|c|c|}
\hline \multirow{2}{*}{$\begin{array}{c}\text { Water quality } \\
\text { indices }\end{array}$} & \multirow{2}{*}{ Units } & \multirow{2}{*}{$\begin{array}{c}\text { Descriptive } \\
\text { statistics }\end{array}$} & \multicolumn{2}{|c|}{ Syrenie Stawy Ponds } \\
\hline & & & Pond No. 1 - inflow area & Pond No. 2 - outflow area \\
\hline Temperature & {$\left[{ }^{\circ} \mathrm{C}\right]$} & $\begin{array}{c}\text { Mean } \pm S D \\
\text { Range } \\
C V \\
\end{array}$ & $\begin{array}{c}10.8 \pm 7.9 \\
0.4-25.2 \\
0.72 \\
\end{array}$ & \begin{tabular}{|c|}
$10.0 \pm 7.4$ \\
$0.4-24.1$ \\
0.74 \\
\end{tabular} \\
\hline $\mathrm{pH}$ & {$[-]$} & $\begin{array}{c}\text { Mean } \pm S D \\
\text { Range } \\
C V \\
\end{array}$ & $\begin{array}{c}7.96 \pm 0.39 \\
7.10-8.60 \\
0.04 \\
\end{array}$ & $\begin{array}{c}7.95 \pm 0.35 \\
7.10-8.40 \\
0.04 \\
\end{array}$ \\
\hline COD-Mn & {$\left[\mathrm{mg} \mathrm{O}_{2} \cdot \mathrm{dm}^{-3}\right]$} & $\begin{array}{c}\text { Mean } \pm S D \\
\text { Range } \\
C V \\
\end{array}$ & $\begin{array}{c}11.0 \pm 2.5 \\
7.2-18.7 \\
0.22 \\
\end{array}$ & $\begin{array}{l}9.8 \pm 1.5 \\
7.2-14.1 \\
0.15 \\
\end{array}$ \\
\hline COD-Cr & {$\left[\mathrm{mg} \mathrm{O}_{2} \cdot \mathrm{dm}^{-3}\right]$} & $\begin{array}{c}\text { Mean } \pm S D \\
\text { Range } \\
C V\end{array}$ & $\begin{array}{c}160 \pm 82 \\
67.4-388.0 \\
0.52 \\
\end{array}$ & $\begin{array}{c}157 \pm 81 \\
64.7-388.0 \\
0.52 \\
\end{array}$ \\
\hline $\mathrm{BOD}_{5}$ & {$\left[\mathrm{mg} \mathrm{O}_{2} \cdot \mathrm{dm}^{-3}\right]$} & $\begin{array}{c}\text { Mean } \pm S D \\
\text { Range } \\
C V\end{array}$ & $\begin{array}{c}3.1 \pm 1.3 \\
0.50-6.60 \\
0.43 \\
\end{array}$ & $\begin{array}{c}3.5 \pm 1.5 \\
0.50-6.60 \\
0.44 \\
\end{array}$ \\
\hline DO & {$\left[\mathrm{mg} \mathrm{O}_{2} \cdot \mathrm{dm}^{-3}\right]$} & $\begin{array}{c}\text { Mean } \pm S D \\
\text { Range } \\
C V\end{array}$ & $\begin{array}{c}3.3 \pm 1.7 \\
0.5-8.4 \\
0.53 \\
\end{array}$ & $\begin{array}{c}3.7 \pm 1.9 \\
0.5-1.9 \\
0.50 \\
\end{array}$ \\
\hline WS & {$[\%]$} & $\begin{array}{c}\text { Mean } \pm S D \\
\text { Range } \\
C V\end{array}$ & $\begin{array}{c}28 \pm 14 \\
4.00-67.00 \\
0.51 \\
\end{array}$ & $\begin{array}{c}31 \pm 15 \\
4.00-67.00 \\
0.50 \\
\end{array}$ \\
\hline $\mathrm{NO}_{3}^{-}$ & {$\left[\mathrm{mg} \mathrm{N}-\mathrm{NO}_{3} \cdot \mathrm{dm}^{-3}\right]$} & $\begin{array}{c}\text { Mean } \pm S D \\
\text { Range } \\
C V\end{array}$ & $\begin{array}{c}0.77 \pm 0.36 \\
0.11-1.75 \\
0.74 \\
\end{array}$ & $\begin{array}{c}0.85 \pm 0.38 \\
0.11-1.75 \\
0.44 \\
\end{array}$ \\
\hline $\mathrm{NO}_{2}^{-}$ & {$\left[\mathrm{mg} \mathrm{N}^{-\mathrm{NO}_{2}} \cdot \mathrm{dm}^{-3}\right]$} & $\begin{array}{c}\text { Mean } \pm S D \\
\text { Range } \\
C V\end{array}$ & $\begin{array}{c}0.28 \pm 0.23 \\
0.104-0.997 \\
0.80 \\
\end{array}$ & $\begin{array}{c}0.17 \pm 0.07 \\
0.083-0.403 \\
0.43 \\
\end{array}$ \\
\hline $\mathrm{NH}_{4}^{+}$ & {$\left[\mathrm{mg} \mathrm{N}^{-\mathrm{NH}_{4}} \cdot \mathrm{dm}^{-3}\right]$} & $\begin{array}{c}\text { Mean } \pm S D \\
\text { Range } \\
C V \\
\end{array}$ & $\begin{array}{c}0.77 \pm 0.33 \\
0.25-1.60 \\
0.42 \\
\end{array}$ & $\begin{array}{c}0.79 \pm 0.28 \\
0.25-1.16 \\
0.35 \\
\end{array}$ \\
\hline $\mathrm{TN}$ & {$\left[\mathrm{mg} \mathrm{N} \cdot \mathrm{dm}^{-3}\right]$} & $\begin{array}{c}\text { Mean } \pm S D \\
\text { Range } \\
C V\end{array}$ & $\begin{array}{c}2.67 \pm 0.60 \\
1.52-3.80 \\
0.22 \\
\end{array}$ & $\begin{array}{c}2.59 \pm 0.65 \\
1.52-3.80 \\
0.25 \\
\end{array}$ \\
\hline SRP & {$\left[\mathrm{mg} \mathrm{P}^{-} \mathrm{PO}_{4} \cdot \mathrm{dm}^{-3}\right]$} & $\begin{array}{c}\text { Mean } \pm S D \\
\text { Range } \\
C V\end{array}$ & $\begin{array}{c}0.23 \pm 0.14 \\
0.04-0.57 \\
0.62 \\
\end{array}$ & $\begin{array}{c}0.24 \pm 0.16 \\
0.05-0.57 \\
0.63 \\
\end{array}$ \\
\hline TP & {$\left[\mathrm{mg} \mathrm{P}^{-} \mathrm{PO}_{4} \cdot \mathrm{dm}^{-3}\right]$} & $\begin{array}{c}\text { Mean } \pm S D \\
\text { Range } \\
C V\end{array}$ & $\begin{array}{c}1.03 \pm 0.46 \\
0.31-2.06 \\
0.44 \\
\end{array}$ & $\begin{array}{c}1.13 \pm 0.65 \\
0.31-3.06 \\
0.57 \\
\end{array}$ \\
\hline TH & {$\left[\mathrm{mg} \mathrm{CaCO}_{3} \cdot \mathrm{dm}^{-3}\right]$} & $\begin{array}{c}\text { Mean } \pm S D \\
\text { Range } \\
C V\end{array}$ & $\begin{array}{c}247 \pm 150 \\
99-681 \\
0.61 \\
\end{array}$ & $\begin{array}{c}289 \pm 129 \\
166-681 \\
0.44 \\
\end{array}$ \\
\hline $\mathrm{Ca}^{2+}$ & {$\left[\mathrm{mg} \mathrm{Ca} \cdot \mathrm{dm}^{-3}\right]$} & $\begin{array}{c}\text { Mean } \pm S D \\
\text { Range } \\
C V\end{array}$ & $\begin{array}{c}73 \pm 29 \\
34-131 \\
0.40\end{array}$ & $\begin{array}{c}85 \pm 25 \\
54-131 \\
0.30\end{array}$ \\
\hline $\mathrm{Cl}^{-}$ & {$\left[\mathrm{mg} \mathrm{Cl} \cdot \mathrm{dm}^{-3}\right]$} & $\begin{array}{c}\text { Mean } \pm S D \\
\text { Range } \\
C V\end{array}$ & $\begin{array}{c}39 \pm 20 \\
22-105 \\
0.53\end{array}$ & $\begin{array}{c}37 \pm 19 \\
22-105 \\
0.53\end{array}$ \\
\hline
\end{tabular}




\begin{tabular}{|c|c|c|c|c|}
\hline \multirow{2}{*}{$\begin{array}{l}\text { Water quality } \\
\text { indices }\end{array}$} & \multirow{2}{*}{ Units } & \multirow{2}{*}{$\begin{array}{c}\text { Descriptive } \\
\text { statistics }\end{array}$} & \multicolumn{2}{|c|}{ Syrenie Stawy Ponds } \\
\hline & & & Pond No. 1 - inflow area & Pond No. 2 - outflow area \\
\hline \multirow{3}{*}{$\mathrm{SO}_{4}^{2-}$} & \multirow{3}{*}[\mathrm{mg}\mathrm{SO}_{4}\cdot\mathrm{dm}^{-3}]{} & Mean $\pm S D$ & $64 \pm 37$ & $62 \pm 37$ \\
\hline & & Range & $33-193$ & $33-193$ \\
\hline & & $C V$ & 0.57 & 0.59 \\
\hline \multirow{3}{*}{ Alkalinity } & \multirow{3}{*}[\mathrm{mg}\mathrm{HCO}_{3}\cdot\mathrm{dm}^{-3}]{} & Mean $\pm S D$ & $222 \pm 50$ & $221 \pm 49$ \\
\hline & & Range & $100-350$ & $100-350$ \\
\hline & & $C V$ & 0.22 & 0.22 \\
\hline \multirow{3}{*}{$\mathrm{Fe}_{\text {tot }}$} & \multirow{3}{*}[\mathrm{mg}\mathrm{Fe}\cdot\mathrm{dm}^{-3}]{} & Mean $\pm S D$ & $0.47 \pm 0.33$ & $0.52 \pm 0.36$ \\
\hline & & Range & $0.05-1.33$ & $0.07-1.33$ \\
\hline & & $C V$ & 0.70 & 0.68 \\
\hline
\end{tabular}

where: $S D$ - standard deviation (uncertainty of measurement), $C V$ - coefficient of variation

On the other hand, higher concentrations of biogenic substances, TP and mineral substances in the outflow compared to the inflow may indicate the influx of pollutants directly into the Ponds, or the periodic release from the bottom sediments under heavy water flow conditions during intensive and sufficiently long precipitation, when by the bypass, which are the Ponds Syrenie Stawy in relation to the Osowka Stream, flows large amounts of water from precipitation.

a)

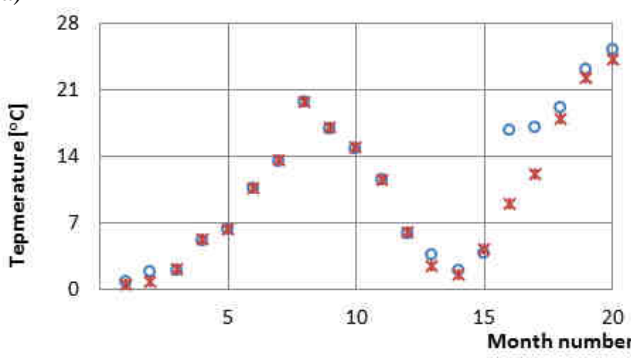

c)

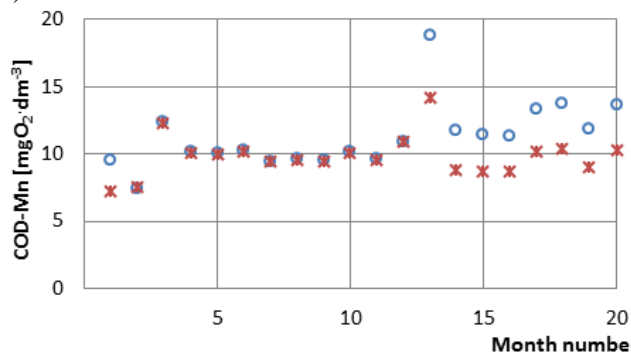

e)

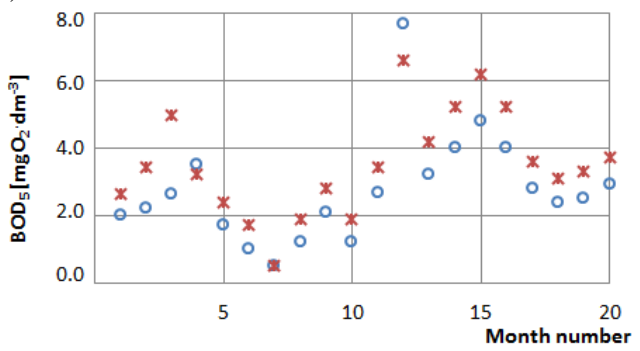

b)

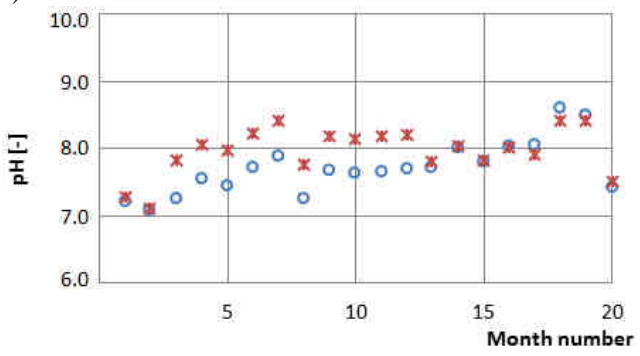

d)

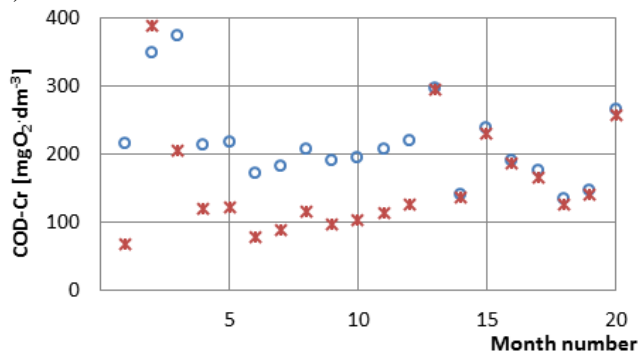

f)

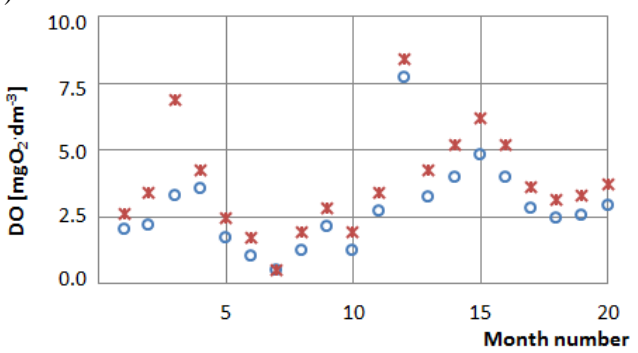


g)

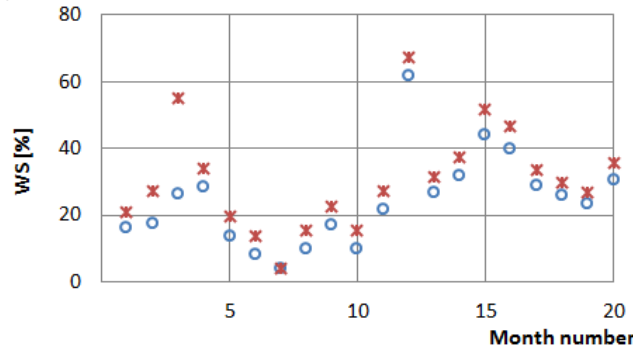

i)

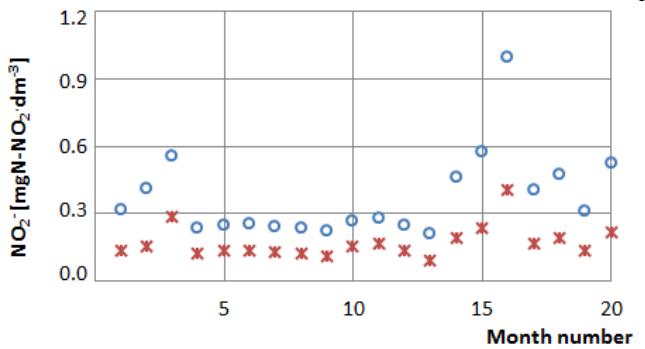

h)

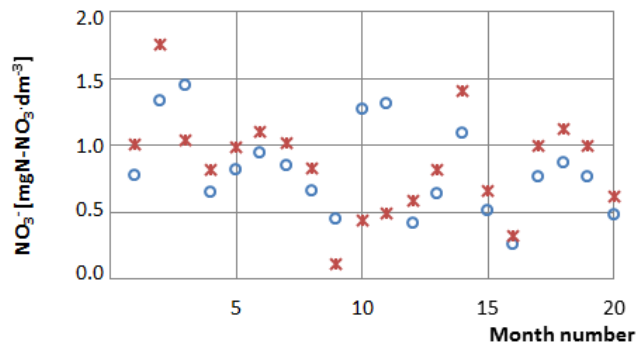

j)

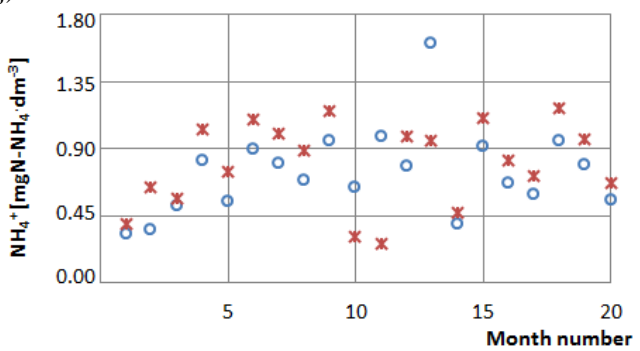

Fig. 2. Comparison of the variability of selected water quality indices of Ponds Syrenie Stawy in the inflow and outflow zone, where: $\circ$ - inflow, $x$ - outflow

a)

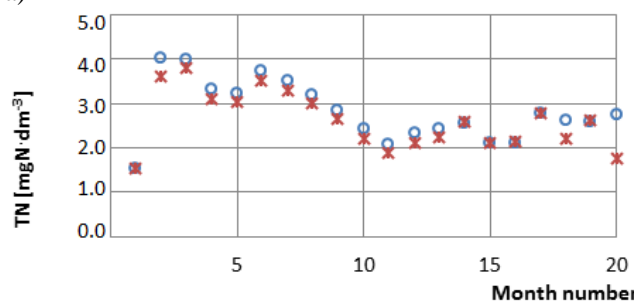

c)

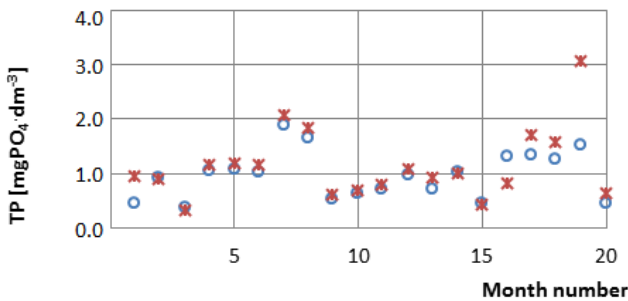

e)

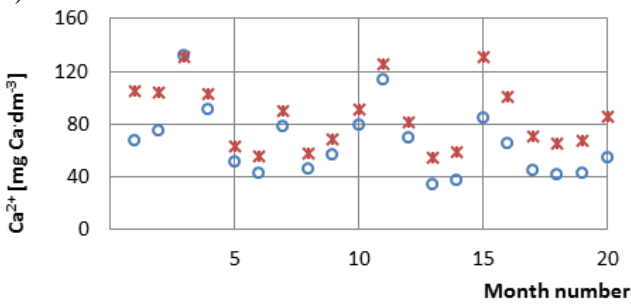

b)

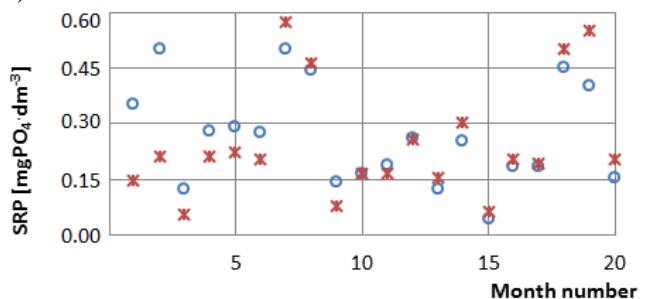

d)

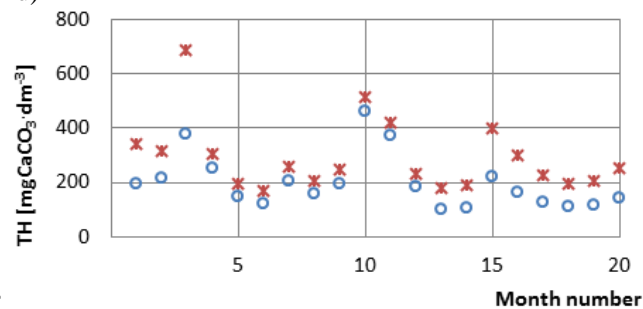

f)

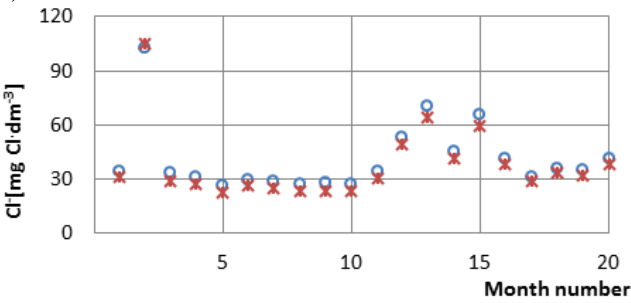


g)

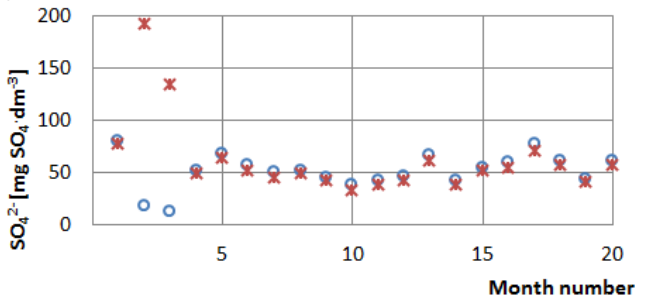

h)

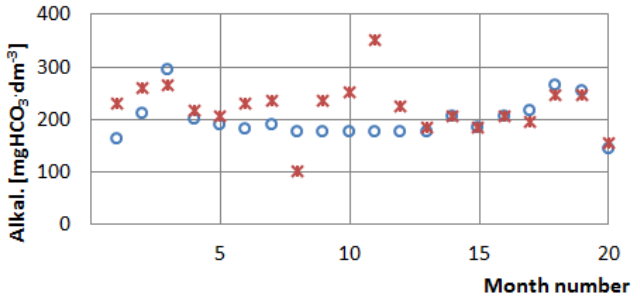

i)

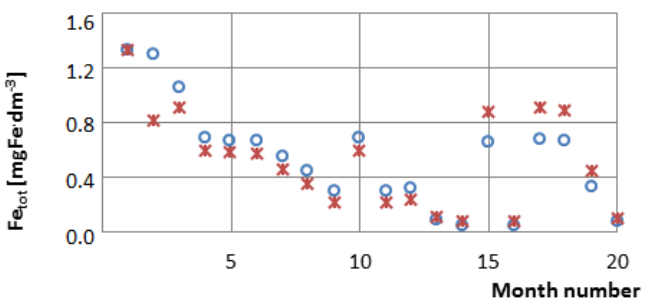

Fig. 3. Comparison of the variability of selected water quality indices of Ponds Syrenie Stawy in the inflow and outflow zone, where: $\circ$ - inflow, $\mathrm{x}$ - outflow

Correlation matrices of selected water quality indices in inflow area of Ponds Syrenie Stawy

Table 3

\begin{tabular}{|c|c|c|c|c|c|c|c|c|c|}
\hline & Temp. & $\mathrm{pH}$ & $\mathrm{COD}-\mathrm{Mn}$ & $\mathrm{COD}-\mathrm{Cr}$ & $\mathrm{BOD}_{5}$ & $\mathrm{DO}$ & $\mathrm{WS}$ & $\mathrm{NO}_{3}{ }^{-}$ & $\mathrm{NO}_{2}{ }^{-}$ \\
\hline Temperature & 1.00 & & & & & & & & \\
\hline $\mathrm{pH}$ & 0.45 & 1.00 & & & & & & & \\
\hline $\mathrm{COD}-\mathrm{Mn}$ & 0.25 & 0.09 & 1.00 & & & & & & \\
\hline $\mathrm{COD}-\mathrm{Cr}$ & -0.07 & -0.45 & 0.61 & 1.00 & & & & & \\
\hline $\mathrm{BOD}_{5}$ & -0.46 & -0.33 & 0.27 & 0.60 & 1.00 & & & & \\
\hline $\mathrm{DO}$ & -0.46 & -0.30 & 0.25 & 0.54 & 0.98 & 1.00 & & & \\
\hline $\mathrm{WS}^{-}$ & -0.26 & -0.21 & 0.45 & 0.62 & 0.93 & 0.95 & 1.00 & & \\
\hline $\mathrm{NO}_{3}{ }^{-}$ & -0.49 & -0.12 & -0.17 & -0.07 & -0.18 & -0.19 & -0.22 & 1.00 & \\
\hline $\mathrm{NO}_{2} \mathrm{NH}_{4}{ }^{+}$ & 0.15 & -0.08 & 0.73 & 0.67 & 0.40 & 0.31 & 0.50 & -0.19 & 1.00 \\
\hline $\mathrm{TN}^{\mathrm{SRP}}$ & 0.08 & 0.32 & 0.07 & -0.07 & -0.18 & -0.12 & -0.15 & 0.01 & -0.35 \\
\hline $\mathrm{TP}$ & 0.04 & 0.01 & -0.05 & 0.07 & -0.29 & -0.24 & -0.22 & 0.58 & -0.31 \\
\hline $\mathrm{TH}^{+2}$ & 0.30 & 0.45 & -0.13 & -0.23 & -0.42 & -0.40 & -0.32 & 0.38 & -0.22 \\
\hline $\mathrm{Ca}^{-}$ & -0.34 & 0.42 & -0.12 & -0.27 & -0.49 & -0.47 & -0.37 & 0.25 & -0.18 \\
\hline $\mathrm{Cl}^{-}$ & -0.32 & -0.12 & -0.66 & -0.37 & 0.07 & 0.12 & -0.03 & 0.02 & -0.47 \\
\hline $\mathrm{SO}_{4}{ }^{2-}$ & -0.30 & -0.59 & 0.21 & 0.51 & 0.14 & 0.11 & 0.16 & 0.42 & 0.22 \\
\hline $\mathrm{Alkalinity}_{\mathrm{Fe}}{ }_{\text {tot }}$ & -0.07 & 0.48 & -0.22 & -0.25 & -0.07 & -0.09 & -0.12 & 0.19 & -0.10 \\
\hline & -0.32 & -0.14 & -0.20 & -0.08 & -0.15 & -0.11 & -0.10 & 0.46 & -0.17 \\
\hline
\end{tabular}

\begin{tabular}{|c|c|c|c|c|c|c|c|c|c|c|}
\hline & $\mathrm{NH}_{4}{ }^{+}$ & TN & SRP & $\mathrm{TP}$ & TH & $\mathrm{Ca}^{2+}$ & $\mathrm{Cl}^{-}$ & $\mathrm{SO}_{4}{ }^{2-}$ & Alkalinity & $\mathrm{Fe}_{\text {tot }}$ \\
\hline Temperat & & & & & & & & & & \\
\hline $\mathrm{pH}$ & & & & & & & & & & \\
\hline COD-M & & & & & & & & & & \\
\hline COD-C & & & & & & & & & & \\
\hline $\mathrm{BOD}_{5}$ & & & & & & & & & & \\
\hline DO & & & & & & & & & & \\
\hline WS & & & & & & & & & & \\
\hline $\mathrm{NO}_{3}^{-}$ & & & & & & & & & & \\
\hline
\end{tabular}




\begin{tabular}{|c|c|c|c|c|c|c|c|c|c|c|}
\hline & $\mathrm{NH}_{4}{ }^{+}$ & $\mathrm{TN}$ & $\mathrm{SRP}$ & $\mathrm{TP}$ & $\mathrm{TH}$ & $\mathrm{Ca}^{2+}$ & $\mathrm{Cl}^{-}$ & $\mathrm{SO}_{4}{ }^{2-}$ & Alkalinity & $\mathrm{Fe}_{\text {tot }}$ \\
\hline $\mathrm{NO}_{2}{ }^{-}$ & & & & & & & & & & \\
\hline $\mathrm{NH}_{4}{ }^{+}$ & 1.00 & & & & & & & & & \\
\hline $\mathrm{TN}$ & 0.28 & 1.00 & & & & & & & & \\
\hline $\mathrm{SRP}$ & 0.17 & 0.27 & 1.00 & & & & & & & \\
\hline $\mathrm{TP}$ & 0.23 & 0.17 & 0.84 & 1.00 & & & & & & \\
\hline $\mathrm{TH}$ & -0.30 & 0.08 & -0.28 & -0.35 & 1.00 & & & & & \\
\hline $\mathrm{Ca}^{2+}$ & -0.31 & 0.03 & -0.30 & -0.33 & 0.97 & 1.00 & & & & \\
\hline $\mathrm{Cl}^{-}$ & -0.02 & -0.33 & -0.18 & -0.26 & -0.36 & -0.22 & 1.00 & & & \\
\hline $\mathrm{SO}_{4}{ }^{2-}$ & -0.03 & 0.34 & -0.24 & -0.07 & -0.10 & 0.01 & 0.31 & 1.00 & & \\
\hline $\mathrm{Alkalinity}-0.20$ & 0.11 & 0.04 & -0.07 & 0.48 & 0.46 & -0.12 & -0.13 & 1.00 & \\
\hline $\mathrm{Fe}_{\text {tot }}$ & -0.12 & 0.29 & -0.11 & -0.04 & 0.46 & 0.46 & -0.14 & 0.51 & 0.39 & 1.00 \\
\hline
\end{tabular}

The bolded values show a statistically significant correlation, at the level of $\alpha=0.05$ between the values of $\mathrm{Cl}^{-}$and the other water quality indices

Table 4

Correlation matrices of selected water quality indices in outflow area of Ponds Syrenie Stawy

\begin{tabular}{|c|c|c|c|c|c|c|c|c|c|}
\hline & Temp. & $\mathrm{pH}$ & $\mathrm{COD}-\mathrm{Mn}$ & $\mathrm{COD}-\mathrm{Cr}$ & $\mathrm{BOD}_{5}$ & $\mathrm{DO}$ & $\mathrm{WS}$ & $\mathrm{NO}_{3}{ }^{-}$ & $\mathrm{NO}_{2}{ }^{-}$ \\
\hline Temperature & 1.00 & & & & & & & & \\
\hline $\mathrm{pH}$ & 0.44 & 1.00 & & & & & & & \\
\hline $\mathrm{COD}-\mathrm{Mn}$ & 0.22 & 0.16 & 1.00 & & & & & & \\
\hline $\mathrm{COD}-\mathrm{Cr}$ & -0.18 & -0.46 & 0.14 & 1.00 & & & & & \\
\hline $\mathrm{BOD}_{5}$ & -0.38 & -0.26 & 0.08 & 0.73 & 1.00 & & & & \\
\hline $\mathrm{DO}$ & -0.43 & -0.23 & 0.17 & 0.67 & 0.96 & 1.00 & & & \\
\hline $\mathrm{WS}^{\mathrm{NO}_{3}{ }^{-}}$ & -0.34 & -0.21 & 0.20 & 0.66 & 0.95 & 0.98 & 1.00 & & \\
\hline $\mathrm{NO}_{2}{ }^{-}$ & -0.32 & -0.06 & -0.05 & 0.09 & -0.16 & -0.17 & -0.16 & 1.00 & \\
\hline $\mathrm{NH}_{4}{ }^{+}$ & 0.28 & 0.47 & 0.17 & -0.07 & -0.11 & -0.07 & -0.02 & -0.03 & -0.28 \\
\hline $\mathrm{TN}^{\mathrm{SRP}}$ & -0.10 & 0.09 & 0.12 & -0.01 & -0.35 & -0.25 & -0.29 & 0.51 & -0.25 \\
\hline $\mathrm{TP}$ & 0.35 & 0.46 & -0.06 & -0.13 & -0.29 & -0.31 & -0.27 & 0.37 & -0.22 \\
\hline $\mathrm{TH}^{2+}$ & 0.29 & 0.38 & -0.01 & -0.27 & -0.43 & -0.43 & -0.42 & 0.40 & -0.43 \\
\hline $\mathrm{Ca}^{-}$ & -0.20 & -0.24 & -0.27 & 0.01 & 0.13 & 0.20 & 0.19 & -0.31 & 0.32 \\
\hline $\mathrm{Cl}^{-}$ & -0.42 & -0.24 & -0.33 & 0.10 & 0.27 & 0.33 & 0.34 & -0.19 & 0.42 \\
\hline $\mathrm{SO}_{4}{ }^{2-}$ & -0.40 & -0.62 & 0.10 & 0.42 & 0.10 & 0.11 & 0.14 & 0.44 & 0.19 \\
\hline $\mathrm{Alkalinity}_{\mathrm{Fe}_{\text {tot }}}$ & -0.06 & 0.41 & -0.06 & -0.26 & -0.22 & -0.18 & -0.20 & 0.18 & 0.06 \\
\hline & -0.25 & -0.15 & 0.01 & -0.10 & -0.24 & -0.16 & -0.12 & 0.42 & 0.10 \\
\hline
\end{tabular}

\begin{tabular}{|c|c|c|c|c|c|c|c|c|c|c|}
\hline & $\mathrm{NH}_{4}^{+}$ & $\mathrm{TN}$ & SRP & $\mathrm{TP}$ & $\mathrm{TH}$ & $\mathrm{Ca}^{2+}$ & $\mathrm{Cl}^{-}$ & $\mathrm{SO}_{4}{ }^{2-}$ & Alkalinity & $\mathrm{Fe}_{\text {tot }}$ \\
\hline \multicolumn{11}{|c|}{ Temperature } \\
\hline \multicolumn{11}{|l|}{$\mathrm{pH}$} \\
\hline \multicolumn{11}{|c|}{ COD-Mn } \\
\hline \multicolumn{11}{|c|}{ COD-Cr } \\
\hline \multicolumn{11}{|l|}{$\mathrm{BOD}_{5}$} \\
\hline \multicolumn{11}{|l|}{ DO } \\
\hline \multicolumn{11}{|l|}{ WS } \\
\hline \multicolumn{11}{|l|}{$\mathrm{NO}_{3}^{-}$} \\
\hline \multicolumn{11}{|l|}{$\mathrm{NO}_{2}^{-}$} \\
\hline $\mathrm{NH}_{4}{ }^{+}$ & 1.00 & & & & & & & & & \\
\hline $\mathrm{TN}$ & 0.20 & 1.00 & & & & & & & & \\
\hline SRP & 0.26 & 0.25 & 1.00 & & & & & & & \\
\hline TP & 0.28 & 0.30 & 0.82 & 1.00 & & & & & & \\
\hline $\mathrm{TH}$ & -0.41 & -0.18 & -0.51 & -0.58 & 1.00 & & & & & \\
\hline $\mathrm{Ca}^{2+}$ & -0.32 & -0.20 & -0.47 & -0.53 & 0.95 & 1.00 & & & & \\
\hline $\mathrm{Cl}^{-}$ & -0.02 & -0.37 & -0.10 & -0.27 & 0.00 & 0.17 & 1.00 & & & \\
\hline
\end{tabular}




\begin{tabular}{|c|c|c|c|c|c|c|c|c|c|c|}
\hline & $\mathrm{NH}_{4}^{+}$ & $\mathrm{TN}$ & $\mathrm{SRP}$ & $\mathrm{TP}$ & $\mathrm{TH}$ & $\mathrm{Ca}^{2+}$ & $\mathrm{Cl}^{-}$ & $\mathrm{SO}_{4}{ }^{2-}$ & Alkalinity & $\mathrm{Fe}_{\text {tot }}$ \\
\hline $\mathrm{SO}_{4}{ }^{2-}$ & -0.06 & 0.25 & -0.25 & -0.06 & 0.00 & 0.12 & 0.22 & 1.00 & & \\
\hline Alkalinity & -0.20 & 0.21 & -0.04 & -0.14 & 0.44 & 0.40 & -0.11 & -0.15 & 1.00 & \\
\hline $\mathrm{Fe}_{\text {tot }}$ & 0.01 & 0.25 & -0.21 & 0.08 & 0.35 & 0.39 & -0.17 & 0.50 & 0.30 & 1.00 \\
\hline
\end{tabular}

The bolded values show a statistically significant correlation, at the level of $\alpha=0.05$ between the values of $\mathrm{Cl}^{-}$and the other water quality indices

During creation artificial neural networks models, the choice of input parameters is a very important aspect. Inadequate parameters will lead to inappropriate network learning, resulting in inadequate results and efficiency of the whole process. The higher the correlation between the independent variables and the dependent variable, the network architecture learning ANN is better. In order to avoid introducing unnecessary variables during the calculations, the correlation between the tested water quality indices and the $\mathrm{Cl}^{-}$ concentration was performed. Correlation coefficients are summarized in Table 3 for water inflow zone, and Table 4 for the water outflow zone of the Ponds Syrenie Stawy. The results show that strong correlations with $\mathrm{Cl}^{-}$values were COD-Cr, $\mathrm{BOD}_{5}, \mathrm{DO}$, WS and $\mathrm{NO}_{2}^{-}$at both sampling stations. Strong correlation loads may indicate that the calculated values of chlorides with ANN should be highly probable [18, 19, 42, 43, 46].

Frequent phenomena during neural network research are the overfitting of training data, leading to a deterioration in the generalizability of the model resulting in undesirable output values. To avoid this phenomenon, the number of hidden layers of neurons was tested $[18,44,48]$. The analyzes were performed using 2, 4, 6, 8 and 10 nodes during the calculation. It was determined that the most likely ANN results are obtained with 5 hidden layers, 1000 iterations and 6 nodes.

In order to check the quality and impact of selected variables on changes in chloride ion concentrations 6 prognostic models were generated for each measurement station. Different input parameters were used for their calculation - each selected water quality indicator independently and collectively using 5 indices together.

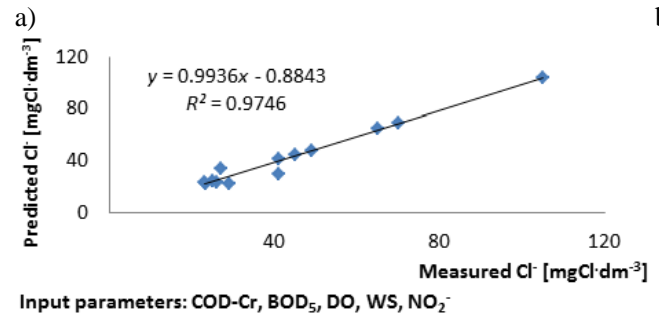

b)
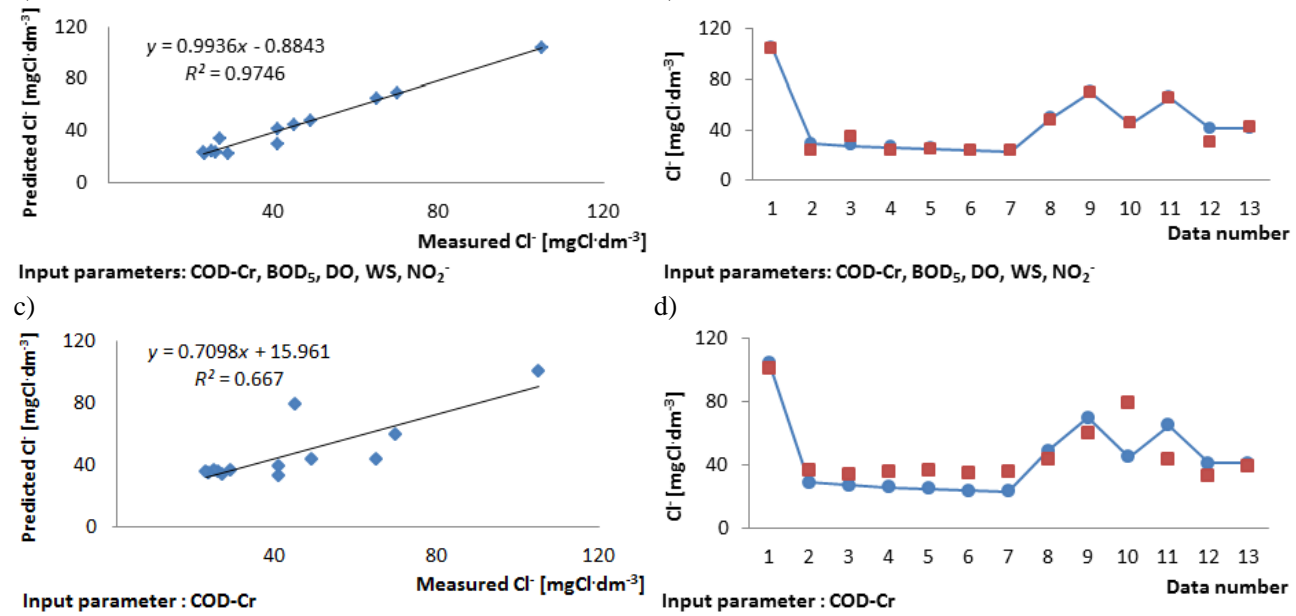

Input parameters: $\mathrm{COD}-\mathrm{Cr}, \mathrm{BOD}_{5}, \mathrm{DO}, \mathrm{WS}, \mathrm{NO}_{2}$

d)

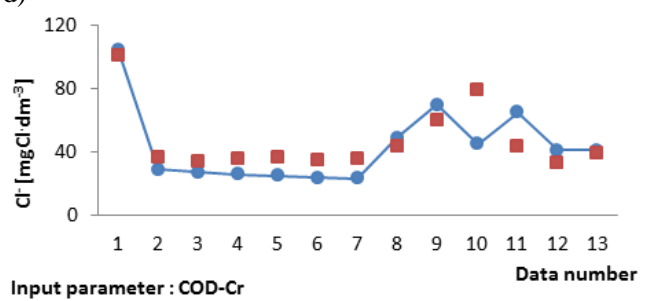


e)

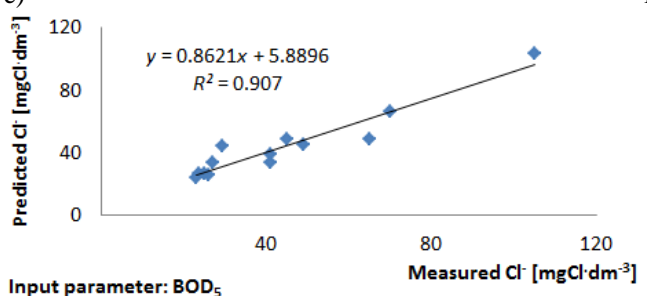

g)

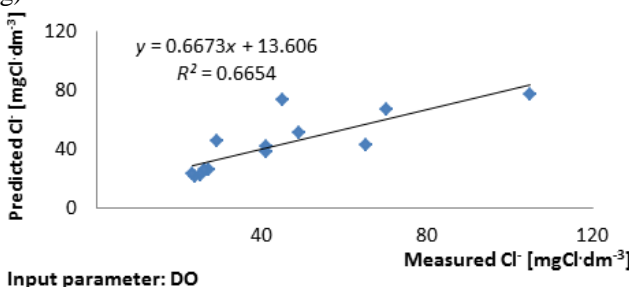

i)

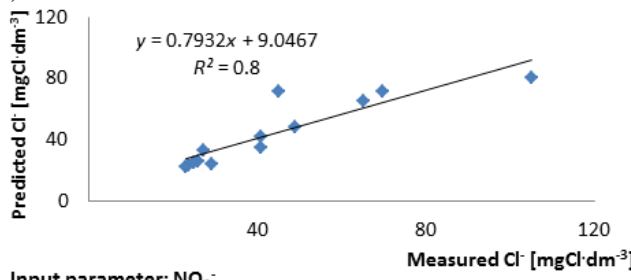

k)

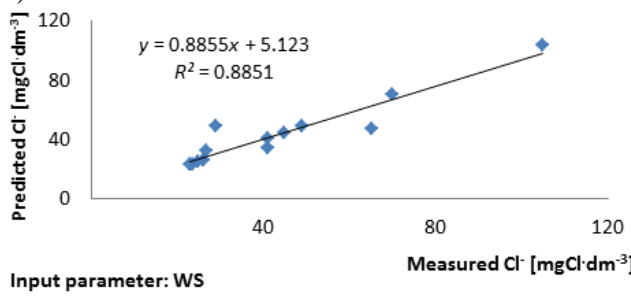

f)

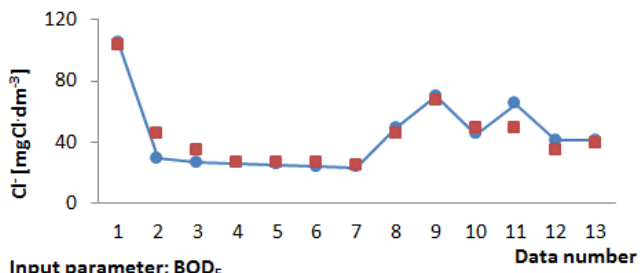

h)

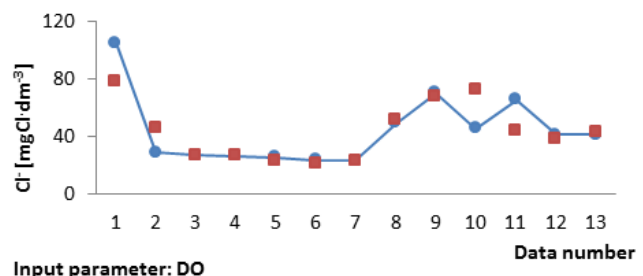

j)

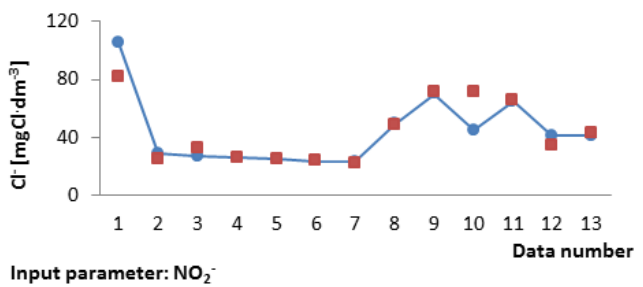

1)

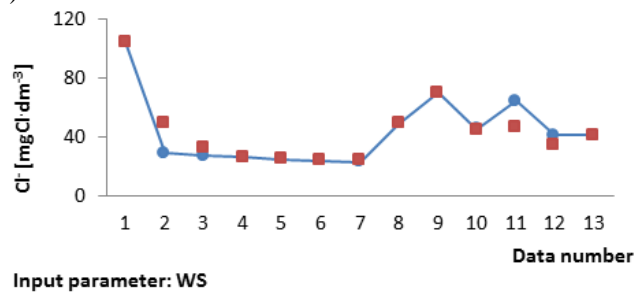

Fig. 4. Results of computed models for each input parameter for the inflow of Syrenie Stawy Ponds

a)

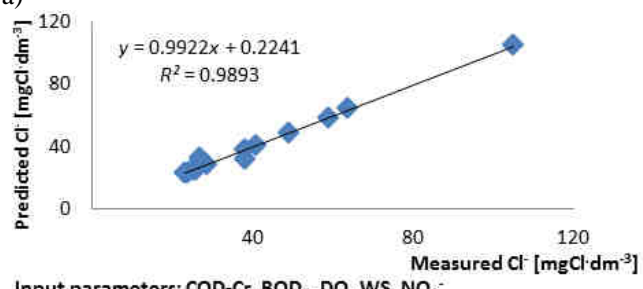

Input parameters: $\mathrm{COD}-\mathrm{Cr}_{1}, \mathrm{BOD}_{5}, \mathrm{DO}$, WS, $\mathrm{NO}_{2}$ b)

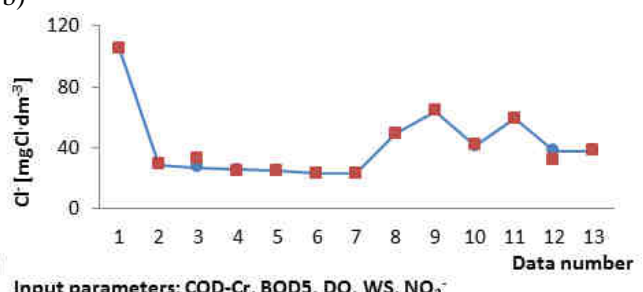


c)

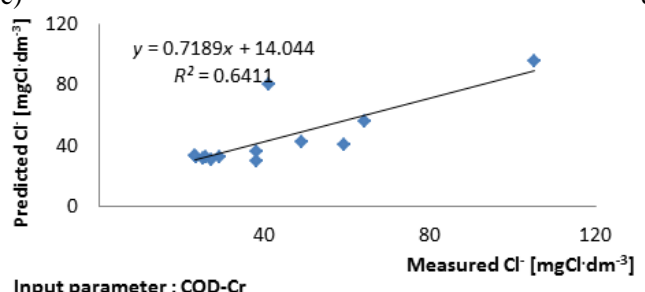

e)

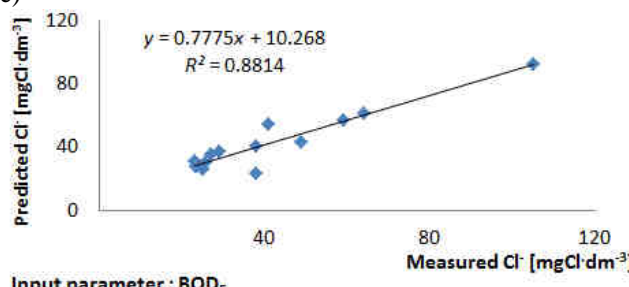

g)

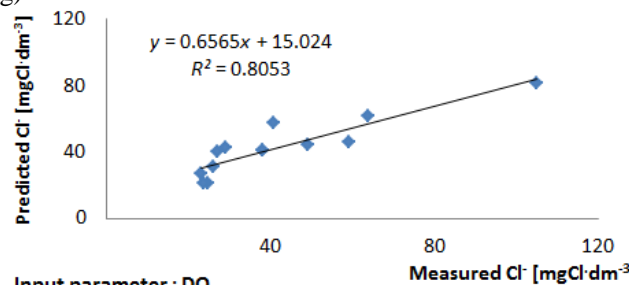

i)

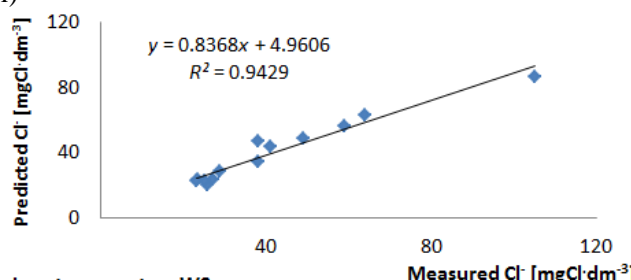

Input parameter : WS

k)

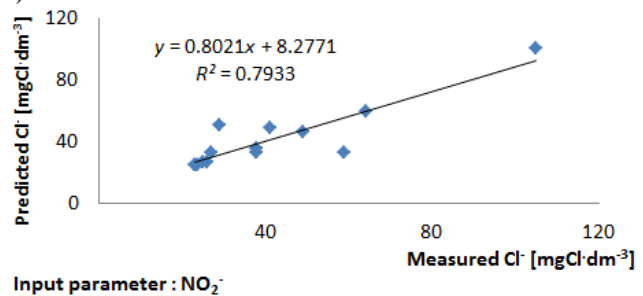

d)

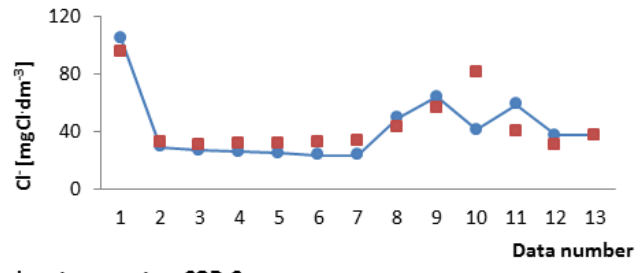

f)

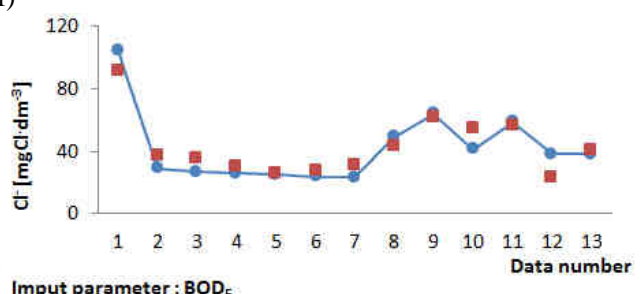

h)

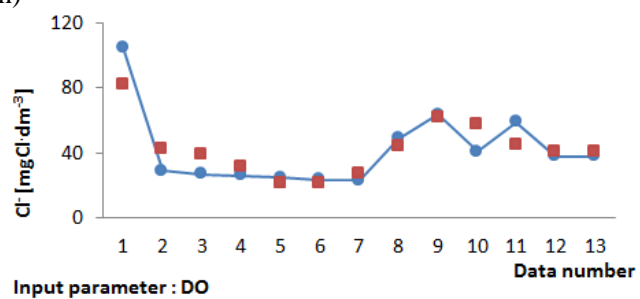

j)

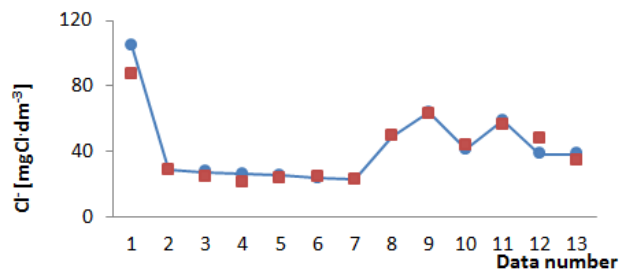

Input parameter : WS

1)

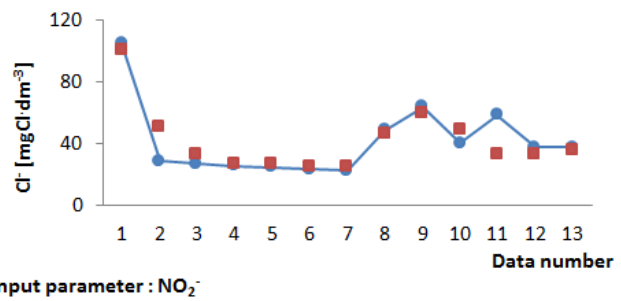

Fig. 5. Results of computed models for each input parameter for the outflow of Syrenie Stawy Ponds

Compatibility of forecasted data with "real" data was determined additionally on the basis of indications of $R^{2}$ coefficient obtained from linear equations (Figs. 4 and 5). The models with the highest values of $R^{2}-0.9746$ and 0.9893 - for the inflow and outflow zone - respectively - were characterized by models where all water quality indices were selected 
as input parameters (i.e. COD-Cr, $\mathrm{BOD}_{5}, \mathrm{DO}, \mathrm{WS}, \mathrm{NO}_{2}^{-}$). The lowest values of the determination coefficient were obtained for single parameter models, in particular COD-Cr $\left(R^{2}=0.667\right)$ and DO $\left(R^{2}=0.6654\right)$ - for the inflow zone and COD-Cr $\left(R^{2}=0.6411\right)$ for the outflow zone. It should be noted that the model based on the WS in the outflow zone was also high $\left(R^{2}=0.9429\right)$ - in comparison to the rest of the tested models.

Tables 5 and 6 show the results of the computational analyzes. It has been shown that the WS determines the highest coefficient of determination $\left(R^{2}=0.887\right)$ among the water quality indices selected for ANN calculations in the water inflow zone to the Ponds. It also showed the lowest MSE and RMSE values, and its mean absolute prediction error was only $10.64 \%$.

Table 5

Evaluation of ANN models for inflow area of ponds Syrenie Stawy

\begin{tabular}{|c|c|c|c|c|c|c|}
\hline \multirow{2}{*}{$\begin{array}{c}\text { Performance } \\
\text { criteria }\end{array}$} & \multicolumn{4}{|c|}{ Input parameter } \\
\cline { 2 - 7 } & COD-Cr & BOD $_{\mathbf{5}}$ & DO & WS & $\mathbf{N O}_{2}^{-}$ & COD-Cr + BOD $_{\mathbf{5}}+\mathbf{D O}+\mathbf{W S}_{\mathbf{2}} \mathbf{N O}_{\mathbf{2}}^{-}$ \\
\hline$M S E$ & 175.68 & 46.76 & 166.40 & 56.92 & 98.86 & 13.99 \\
\hline$R M S E$ & 13.25 & 6.83 & 12.89 & 7.54 & 9.94 & 3.74 \\
\hline$M A P E$ & 30.51 & 13.51 & 16.95 & 10.64 & 11.56 & 7.05 \\
\hline$R^{2}$ & 0.799 & 0.819 & 0.666 & 0.887 & 0.788 & 0.999 \\
\hline
\end{tabular}

Table 6

Evaluation of ANN models for outflow area of ponds Syrenie Stawy

\begin{tabular}{|c|c|c|c|c|c|c|}
\hline \multirow{2}{*}{$\begin{array}{c}\text { Performance } \\
\text { criteria }\end{array}$} & \multicolumn{4}{|c|}{ Input parameter } \\
\cline { 2 - 7 } & COD-Cr & BOD $_{\mathbf{5}}$ & DO & WS & NO $_{\mathbf{2}}^{-}$ & COD-Cr + BOD $_{\mathbf{5}}+\mathbf{D O}_{\mathbf{+}} \mathbf{W S}_{\mathbf{+}} \mathbf{N O}_{\mathbf{2}}^{-}$ \\
\hline$M S E$ & 174.26 & 60.59 & 102.11 & 34.14 & 95.04 & 4.94 \\
\hline$R M S E$ & 13.20 & 7.78 & 10.10 & 5.84 & 9.75 & 2.22 \\
\hline$M A P E$ & 26.55 & 18.38 & 20.74 & 8.66 & 17.72 & 3.42 \\
\hline$R^{2}$ & 0.781 & 0.687 & 0.532 & 0.854 & 0.731 & 0.995 \\
\hline
\end{tabular}

Analysis of the efficiency and goodness of fit among the water quality indices in the Ponds Syrenie Stawy outflow zone has also shown that the best performing indicator is also the WS of $R^{2}$ of 0.854 and MAPE on level of $8.66 \%$.

For both measurement stations, the worst predicted indicator based on the determination coefficient of 0.666 in the water inflow zone and 0.532 in the water outflow zone, respectively, was the concentration of dissolved oxygen (DO). On the other hand, while analyzing the mean absolute prediction error $(M A P E)$, despite the high determination coefficient - ca. $R^{2}=0.8$ for both measurement stations - COD-Cr - dependent models had the highest prediction error.

The best calculated ANN models are models with five input parameters, which clearly coincides with the other papers $[18,44]$. The determination coefficients obtained were 0.999 and 0.995 in the inflow and outflow zone respectively. Despite a slightly higher $R^{2}$ in the inlet zone, the rest of the performance and model fitting criteria were better for the forecasts of chlorine concentrations in the outflow zone from Ponds Syrenie Stawy, in particular $M S E=4.94, R M S E=2.22$ and $M A P E$ of only $3.42 \%$

In order to finally compare the ANN results, the discrepancy ratio coefficients $\left(D_{r}\right)$ summarized in Table 7 and Figure 6 were calculated. Table 7 presents the results of the basic statistical analysis, which shows that the divergence of the computed models result for the water inflow zone is lower than for the outflow zone of the Ponds Syrenie Stawy. 
At the same time, following the scattering of the results in Figure 6, it can be seen that the $D_{r}$ factor is most similar to the perfect data distribution in the inflow zone, where - in the outflow area, the discrepancy is greater and in a case of $D_{r}$ over $50 \%$ has been reported. However, such a decomposition could be found in the literature [18, 49-51].

Table 7

Alignment of ANN models as coefficients of discrepancy of results

\begin{tabular}{|c|c|c|c|c|c|}
\hline \multirow{2}{*}{ ANN models for } & \multicolumn{5}{|c|}{ Discrepancy ratio } \\
\cline { 2 - 6 } & $\boldsymbol{D}_{\boldsymbol{r}_{\min }}$ & $\overline{\boldsymbol{D}_{\boldsymbol{r}}}$ & $\boldsymbol{D}_{\boldsymbol{r}_{\max }}$ & $\boldsymbol{S} \boldsymbol{D}$ & $\boldsymbol{C V}$ \\
\hline Inflow & 0.748 & 0.973 & 1.251 & 0.12 & 0.12 \\
\hline Outflow & 0.670 & 1.171 & 1.766 & 0.34 & 0.29 \\
\hline
\end{tabular}

a)

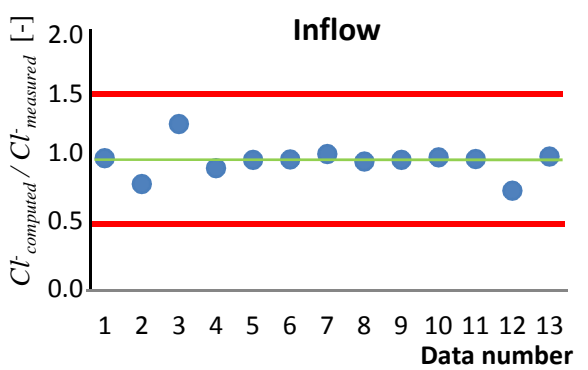

b)

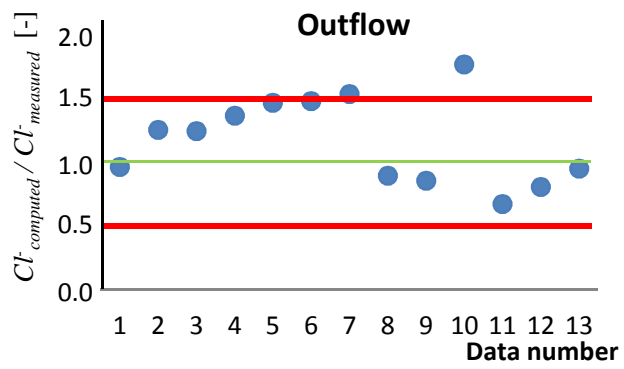

Fig. 6. The discrepancy ratios of best computed ANN models for ponds Syrenie Stawy in inflow and outflow area, where (-) shows 50\% error bands, and (-) perfect fitting points

\section{Conclusions}

The possibility of using artificial neural networks to estimate changes in chloride ion concentrations in urban ponds was investigated on the example of Ponds Syrenie Stawy in Szczecin (NW-Poland). Based on the five selected water quality indices used as input parameters for the ANN model generation, satisfactory mathematical models with high predictive power and low predictive error values were obtained. It has been shown that out of the five selected water quality indices, the parameter that creates the ANN models with the best prognostic coefficient values is water saturation both in the inflow zone and in the outflow zone of water to and from the ponds Syrenie Stawy.

\section{References}

[1] Hill MJ, Biggs J, Thornhill I, Briers RA, Gledhill DG, White JC, Hassall C. Urban ponds as an aquatic biodiversity resource in modified landscapes. Glob Change Boil. 2017;23(3):986-999. DOI: 10.1111/gcb.13401.

[2] Song K, Winters C, Xenopoulos MA, Marsalek J, Frost PC. Phosphorus cycling in urban aquatic ecosystems: connecting biological processes and water chemistry to sediment $\mathrm{P}$ fractions in urban stormwater management ponds. Biogeochemistry. 2017;132(1-2):203-212. DOI: 10.1007/s10533-017-0293.

[3] Olguín EJ, Sánchez-Galván G, Melo FJ, Hernández VJ, González-Portela RE. Long-term assessment at field scale of Floating Treatment Wetlands for improvement of water quality and provision of ecosystem services in a eutrophic urban pond. Sci Total Environ. 2017;584:561-571. DOI: 10.1016/j.scitotenv.2017.01.072.

[4] Angyal Z, Sárközi E, Gombás Á, Kardos L. Effects of land use on chemical water quality of three small streams in Budapest. O Geosci. 2016; 8(1):133-142. DOI: 10.1515/geo-2016-0012.

[5] Gutchess K, Jin L, Lautz L, Shaw SB, Zhou X, Lu Z. Chloride sources in urban and rural headwater catchments, central New York. Sci Total Environ. 2016; 565:462-472. DOI: 10.1016/j.scitotenv.2016.04.181. 
[6] Corsi SR, De Cicco LA, Lutz MA, Hirsch RM. River chloride trends in snow-affected urban watersheds: increasing concentrations outpace urban growth rate and are common among all seasons. Sci Total Environ. 2015;508:488-497. DOI: 10.1016/j.scitotenv.2014.12.012.

[7] Thornton JA, Slawski TM, Lin H. Salinization: the ultimate threat to temperate lakes, with particular reference to Southeastern Wisconsin (USA). Chin J Oceanol Limnol. 2015;33(6):1461-1475. DOI: 10.1007/s00343-015-4368-3.

[8] Dojlido J, Best GA. Chemistry of Water and Water Pollution. New York: Ellis Horwood Limited. 1993; $364 \mathrm{pp}$.

[9] Corsi SR, Graczyk DJ, Geis SW, Booth NL, Richards KD. A fresh look at road salt: aquatic toxicity and water-quality impacts on local, regional, and national scales. Environ Sci Technol. 2010;44(19):7376-7382. DOI: 10.1021/es101333u.

[10] Cieśliński R, Piekarz J, Zieliński M. Groundwater supply of lakes: the case of Lake Raduńskie Górne (northern Poland, Kashubian Lake District). Hydrol Sci J. 2016;61(13):2427-2434. DOI: 10.1080/02626667.2015.1112903.

[11] Rhodes AL, Guswa AJ. Storage and release of road-salt contamination from a calcareous lake-basin fen, western Massachusetts, USA. Sci Total Environ. 2016; 545:525-545. DOI: 10.1016/j.scitotenv.2015.12.060.

[12] Schweiger AH, Audorff V, Beierkuhnlein C. Salt in the wound: The interfering effect of road salt on acidified forest catchments. Sci Total Environ. 2015; 532:595-604.DOI: 10.1016/j.scitotenv.2015.06.034.

[13] Sibert RJ, Koretsky CM, Wyman DA. Cultural meromixis: Effects of road salt on the chemical stratification of an urban kettle lake. Chem Geol. 2015; 395:126-137. DOI: 10.1016/j.chemgeo.2014.12.010.

[14] Novotny EV, Murphy D, Stefan HG. Increase of urban lake salinity by road deicing salt. Sci Total Environ. 2008;406(1):131-144. DOI: 10.1016/j.scitotenv.2008.07.037.

[15] Vitale SA, Robbins GA, McNaboe LA. Impacts of road salting on water quality in fractured crystalline bedrock. J Environ Qual. 2017; 46(2):288-294. DOI: 10.2134/jeq2016.10.0411.

[16] Safi SK, White AK. Short and long-term forecasting using artificial neural networks for stock prices in Palestine: a comparative study. Electron J Appl Statist Anal. 2017;10(1):14-28. DOI: 10.1285/i20705948v10n1p14.

[17] Moghim S, Bras RL. Bias correction of climate modeled temperature and precipitation using artificial neural networks. J Hydrometeorol. 2017. DOI: 10.1175/J HM-D-16-0247.1.

[18] Sengorur DEB, Koklu R. Modeling biological oxygen demand of the Melen River in Turkey using an artificial neural network technique. J Environ Manage. 2009;90(2):1229-1235. DOI: 10.10116/j.jevman.2008.06.004.

[19] Dastorani MT, Afkhami H, Sharifidarani H, Dastorani M. Application of ANN and ANFIS models on dryland precipitation prediction (case study: Yazd in Central Iran). J Appl Sci. 2010;10(20):2387-2394. DOI: 10.1007/s10661-009-1012-8.

[20] Zhang L, Zhang GX, Li RR. Water quality analysis and prediction using hybrid time series and neural network models. J Agri Sci Tech. 2016;18(4):975-983. http://journals.modares.ac.ir/ article_14707_3d8651ca0390d757de18ce6c8528b1a8.pdf.

[21] Mulia IE, Asano T, Tkalich P. Retrieval of missing values in water temperature series using a data-driven model. Ear Sci Inf. 2015;8(4):787-798. DOI: 10.1007/s12145-015-0210-x.

[22] Heddam S, Lamda H, Filali S. Predicting effluent biochemical oxygen demand in a wastewater treatment plant using generalized regression neural network based approach: a comparative study. Environ Process. 2016;3(1):153-165. DOI: 10.1007/s40710-016-0129-3.

[23] Li YL, Zhang Q, Werner AD, Yao J. Investigating a complex lake-catchment-river system using artificial neural networks: Poyang Lake (China). Hydrol Res. 2015;46(6):912-928. DOI: 10.2166/nh.2015.150.

[24] Cole JC, Maloney KO, Schmid M, McKenna JE. Developing and testing temperature models for regulated systems: A case study on the Upper Delaware River. J Hydrol. 2014;519:588-598. DOI: 10.1016/j.jhydrol.2014.07.058.

[25] Hadzima-Nyarko M, Rabi A, Šperac M. Implementation of artificial neural networks in modeling the waterair temperature relationship of the River Drava. Water Resour Manage. 2014; 28(5):1379-1394. DOI: 10.1007/s11269-014-0557-7.

[26] Rabi A, Hadzima-Nyarko M, Šperac M. Modelling river temperature from air temperature: case of the River Drava (Croatia). Hydrolo Sci J. 2015; 60(9):1490-1507. DOI: 10.1080/02626667.2014.914215.

[27] Fajčíková K, Stehlíková B, Cvečková V, Rapant S. Application of artificial neural network in medical geochemistry. Environ Geochem Health. 2017;39(6):1513-1529. DOI: 10.1007/s10653-017-9944-3.

[28] Salami SE, Ehteshami M. Application of artificial neural networks to estimating DO and salinity in San Joaquin River basin. Desal Water Treat. 2016;57(11):4888-4897. DOI: 10.1080/19443994.2014.995713. 
[29] Zheng F, Wan Y, Song K, Sun D, Hedgepeth M. Reconstructing input for artificial neural networks based on embedding theory and mutual information to simulate soil pore water salinity in tidal floodplain. Water Resource Res. 2016;52:511-532 DOI: 10.1002/2014WR016875.

[30] Chen W, Liu W, Huang W, Liu H. Prediction of salinity variations in a tidal estuary using artificial neural network and three-dimensional hydrodynamic models. Comput Water Ener Environ Eng. 2017;6:107-128. DOI: $10.4236 /$ cweee.2017.61009.

[31] Rath JS, Hutton PH, Chen L, Roy SB. A hybrid empirical-Bayesian artificial neural network model of salinity in the San Francisco Bay-Delta estuary. Environ Model Soft. 2017;93:193-208. DOI: 10.1016/j.envsoft.2017.03.022.

[32] Dandy G. Use of artificial neural networks to forecast salinity in rivers. Artificial Neural Networks in Water Supply Engineering. ASCE. 2005;107-137.

[33] Poleszczuk G, Miller T, Tokarz M. Ponds Syrenie Stawy in Szczecin - changes of selected water quality chemical indices. Chem Didact Ecol Metrol. 2015;20(1-2):85-96 DOI: 10.1515/cdem-2015-0009.

[34] Miller T, Tokarz M, Poleszczuk G. Stream Osowka in Szczecin - chemometric analysis of water quality indices in winter season. Acta Biol. 2014;21:91-104. http://www.wb.usz.edu.pl/attachments/article/657/ 6-Miller.pdf.

[35] Poleszczuk G, Wawrzyniak W, Miller T, Tokarz M, Gasperowicz A, Adamczyk D. Syrenie Stawy in Szczecin - a comparison of the quality of waters in the winter and spring in 1994-1995, 1999-2000, 2006-2007 and 2014. In: Wawrzyniak W, Zaborowski T, editors. Ecology of Borderlands. Monography. Gorzow Wlkp.-Poznan: IBEN. 2014;122-137. DOI: 10.13140/2.1.2179.2006.

[36] Sammel A. Skład chemiczny osadów dennych zbiorników wodnych Syrenie Stawy aglomeracji szczecińskiej i możliwości ich wykorzystania. Inż Środ. 2015; 157(37):53-60.

[37] APHA. Standard methods for the examination of water and wastewater. Washington: American Public Health Association. 2012; 1800pp.

[38] Fahimi F, Yaseen ZM, El-shafie A. Application of soft computing based hybrid models in hydrological variables modeling: a comprehensive review. Theor Appl Climatol. 2017;128(3):875-903. DOI: 10.1007/s00704-016-1735-8.

[39] Chen XY, Chau KW, Wang WC. A novel hybrid neural network based on continuity equation and fuzzy pattern-recognition for downstream daily river discharge forecasting. J Hydroinf. 2015; 17(5):733-744. DOI: 10.2166/hydro.2015.095.

[40] Yaseen ZM, El-Shafie A, Afan HA, Hameed M, Mohtar WHMW, Hussain A. RBFNN versus FFNN for daily river flow forecasting at Johor River, Malaysia. Neural Comput Applic. 2016; 27(6):1533-1542. DOI: 10.1007/s00521-015-1952-6.

[41] Yaseen ZM, El-Shafie A, Jaafar O, Afan HA, Sayl KN. Artificial intelligence based models for stream-flow forecasting: 2000-2015. J Hydrology. 2015;530:829-844. DOI: 10.1016/j.jhydrol.2015.10.038.

[42] Talaee PH. Multilayer perceptron with different training algorithms for streamflow forecasting. Neural Comput Applic. 2014;24(3-4):695-703. DOI: 10.1007/s00521-012-1287-5.

[43] Trichakis IC, Nikolos IK, Karatzas GP. Optimal selection of artificial neural network parameters for the prediction of a karstic aquifer's response. Hydrol Proces. 2009; 23(20):2956-2969. DOI: 10.1002/hyp.7410.

[44] Piotrowski AP, Napiorkowski JJ. A comparison of methods to avoid overfitting in neural networks training in the case of catchment runoff modelling. J Hydrol. 2013;476:97-111. DOI: 10.1016/j.jhydrol.2012.10.019.

[45] Banhatti AG, Deka PC. Effects of data pre-processing on the prediction accuracy of artificial neural network model in hydrological time series. Urban Hydrol Wat Manag Soc Econ Aspec. 2016;265-275. DOI: 10.1007/978-3-319-40195-9_21.

[46] Li X, Maier HR, Zecchin AC. Improved PMI-based input variable selection approach for artificial neural network and other data driven environmental and water resource models. Environ Model Soft. 2015;65:15-29. DOI: 10.1016/j.envsoft.2014.11.028.

[47] Kasiviswanathan, KS, Sudheer KP, He J. Quantification of Prediction Uncertainty in Artificial Neural Network Models. In Artificial Neural Network Modelling Springer International Publishing. 2016;145-159. DOI: $10.1007 / 978-3-319-28495-8 \_8$.

[48] Piotrowski AP, Napiorkowski MJ, Napiorkowski JJ, Osuch M. Comparing various artificial neural network types for water temperature prediction in rivers. J Hydrol. 2015;529:302-315. DOI: 10.1016/j.jhydrol.2015.07.044.

[49] Antonopoulos VZ, Georgiou PE, Antonopoulos ZV. Dispersion coefficient prediction using empirical models and ANNs. Environ Proces. 2015;2(2):379-394. DOI: 10.1007/s40710-015-0074-6

[50] Kashefipour SM, Falconer RA. Longitudinal dispersion coefficients in natural channels. Water Res. 2002; 36(6):1596-1608. DOI: 10.1016/S0043-1354(01)00351-7.

[51] Seo IW, Cheong TS. Predicting longitudinal dispersion coefficient in natural streams. J Hydraulic Eng. 1998;124(1):25-32. DOI: 10.1061/(ASCE)0733-9429(1998)124:1(25). 